Commun. math. Phys. 12, 80-90 (1969)

\title{
Symmetries Induced by Conserved Vector Currents in the Theory of a Scalar Field
}

\author{
JAN T. ŁoPUSZAŃSKI \\ Institute of Theoretical Physics, University of Wrocław, Wrocław \\ Institute of Low Temperature Physics and Structural Research \\ Polish Academy of Sciences, Wrocław
}

Received September 19, 1968

\begin{abstract}
Let us consider a quantum theory of one scalar, real, local, Poincaré covariant field $A(x)$ with the restricted spectrum condition (massive one particle states and a unique vacuum). The asymptotic fields $A_{\text {in }}(x)$ are assumed to be irreducible. Our conjecture is that under some technical assumptions the "charge" of every real, hermitean, locally conserved, Poincaré covariant quantum (pseudo) vector field $j_{\mu}(x)$ relatively local to $A(x)$, appearing in this theory-vanishes. This means that in a theory of one scalar, real field with a massive particle one can not expect to get symmetry groups induced by conserved (pseudo) vector currents, only by global, selfadjoint, Poincaré invariant generators.

Our arguments can be easily extended to a theory of one complex scalar field, in this case the only symmetry transformation induced by a current can be the gauge transformation.

We prove also that under very weak assumptions two fields related to each other by a unitary (or similarity) transformation are equal barring some patological cases.
\end{abstract}

\section{Introduction}

In many papers concerned with the symmetry problems in quantum field theory (e.g. Goldstone's Theorem and miscellaneous topics related to it) an algebra of quasilocal observables as well as a conserved vector current $j_{\mu}(x)$ local with respect to the elements of the algebra and to itself are under investigation (see e.g. [1-3]).

It is well known that the necessary condition to get a properly defined "charge" (which in turn gives rise to a one parameter symmetry group of the theory under consideration) is the local conservation of the current $j_{\mu}(x)$. If the energy-momentum spectrum has a mass gap then it is also a sufficient condition (no - so called - "Goldstone's particles"). The theorems proved up to now state the existence of the "charge". They do not, however, exclude the case that the "charge" vanishes. Of course, such a vanishing "charge" does not give rise to a symmetry.

It is of some interest to learn are we able at all to construct a conserved local current $j_{\mu}(x)$ out of a scalar, local, irreducible quantum field $A(x)$ in such a way that $j_{\mu}(x)$ is local with respect to $A(x)$, transforms 
under the same Poincaré group representation as $A(x)$ and gives rise to a conserved nonvanishing "charge".

Our conjecture is that this can not be done for a real field (the complex field admits a gauge transformation) provided there is a discrete one particle mass different from zero in the energy-momentum spectrum. Loosely speaking this would mean that in a theory of one scalar real field with one massive particle one can not expect to get any one parameter symmetry group induced by a conserved (pseudo) vector current. The only symmetry groups we may find are the global symmetries generated by self-adjoint quantities invariant under the Poincaré transformations.

In $\S 2$ we list our basic assumptions. We prove that two real fields $A$ and $B=V A V^{+}$(where $V$ is unitary with a nonvanishing v.e.v.) transforming under the same representation of the Poincaré group, almost local with respect to each other are equal, barring some patological cases.

In $\S 3$ plausible arguments in favour of our main conjecture are presented.

In $\S 4$ we extend our arguments to a complex scalar field and learn that the only admissible candidate for a symmetry induced by a current is the gauge transformation.

In $\S 5$ we show that a similarity transformation which links two real fields together can be practically always replaced by a unitary transformation, a result which was already stated in a different context by other authors [4].

Finally in $\S 6$ we discuss some examples referring to our main conjecture.

\section{Assumptions and Statement}

The relevant assumptions are the usual ones, namely.

i) The Poincare group is unitarily implemented in the Hilbert space $\mathscr{H}$. We denote the representation of $(\Lambda, a)$ by $U(\Lambda, a)$. The spectrum of $P^{2}$, where $\exp (i P a)=U(I, a)$, consists of two discrete points $\mu^{2}=0$ (corresponding to the single vacuum state $\Omega$ ) and $m^{2} \neq 0$ (corresponding to one particle states) as well as out of a continuous part, say $\left(4 m^{2}, \infty\right)$ (scattering states).

ii) There exists a local scalar real field $A(x)$ transforming under the Poincaré transformation according to

$$
U(\Lambda, a) A(x) U(\Lambda, a)^{-1}=A(\Lambda x+a) .
$$

We assume for simplicity reasons that

$$
(\Omega, A(x) \Omega)=0 \text {. }
$$

Assumptions i) and ii) imply the existence of free asymptotic fields $A_{\text {in }}(x)$ and $A_{\text {out }}(x)[5]$ provided.

$$
E\left(p^{2}=m^{2}, p_{0}>0\right) A(f) \Omega \neq 0 \quad \text { (see [6]) }
$$

6 Commun.math. Phys., Vol. 12 
where $E\left(p^{2}=m^{2}, p_{0}>0\right)$ is the projection on the subspace spanned by one particle states, $A(f)=\int A(x) f(x) d x, f(x) \in \mathscr{S}_{4}$.

We assume in addition that

iv) The asymptotic field $A_{\text {in }}(x)$ is irreducible. This implies that also $A_{\text {out }}(x)=\Theta A_{\text {in }}(x) \Theta[\Theta$ is the TCP operator of $A(x)]$ as well as $A(x)$ are irreducible.

Under these assumptions we have the

Statement. Assume there is such a unitary operator $V$ that

$$
(\Omega, V \Omega) \neq 0
$$

and the field

$$
B(x) \equiv V A(x) V^{+}
$$

transforms under the same representation of the Poincaré group as $A(x)$ and is almost local with respect to $A(x)$ i.e.

$$
R^{N}\left\langle\prod_{j=1}^{s} C_{l_{j}}\left(x_{j}\right)\right\rangle_{T} \underset{R \rightarrow \infty}{\longrightarrow} 0 .
$$

$N$ any real integer, $T$ stands for "truncated" where $C_{l,}$ are either $A$ - or $B$-fields and $x_{j}$ 's are clustered. Then a) $V$ is a multiple of identity, or b) the theory is such that the particle number for the field $A$ is conserved, i.e. $N^{\text {in }}=N^{\text {out }}=N$; in this case

$$
V=\exp \{i(\pi N+b)\}, \quad b \text { real number . }
$$

Proof. From the fact that $A$ and $B$ transform under the same representation of the Poincaré group, $A(x)$ is irreducible and (4) holds follows that $V$ commutes with $U(\Lambda, a)$. Then

$$
V \Omega=e^{i b} \Omega, \quad b \text { real number . }
$$

From the assumptions above follows that ([6-8]) the asymptotic fields $A_{\text {in }}(x)$ and $B_{\text {in }}(x)$ exist and are related to each other by

$$
A_{\text {in }}(x)= \pm \underset{\text { out }}{\text { out }}(x) .
$$

Since the mapping induced by $V$ is a global one relation (5) can be extended also to asymptotic fields, viz.

$$
B_{\text {in }}(x)=V A_{\text {out }}(x) V^{+} .
$$

Taking into account the irreducibility of the asymptotic fields as well as (8), (9) and (10) $V$ can be uniquely defined: in case $A_{\text {in }}(x)=\underset{\text { out }}{\text { out }}$ ( $V=\exp (i b)$, in case $A_{\text {in }}(x)=-\underset{\text { out }}{\text { out }}$ in $(x) V$ is given by $(7 \mathrm{~b})$ provided (7a) is fulfilled [the case $\pi N_{\text {in }}=(2 n+1) \pi N_{\text {out }}$ is excluded due to the stability of the one particle states]..$^{\prime}$

$1^{\prime}$ Although in the framework of axiomatic field theory we are not aware of any proof that $N_{\text {in }}=N_{\text {out }}$ implies $S=1$ ( $S$ is the scattering matrix) there are strong indications that it is so (see e. g. S. Ø. AKs, J. Math. Phys. 6, 516 (1965)). 


\section{A Conjecture Concerning the Case of One Real Field}

Since our main goal is to construct a (pseudo) vector field $j_{\mu}(x)$ which gives rise to a symmetry operation we have to add some new assumptions to the previously stated, namely.

v) There exists a real (pseudo) vector field $j_{\mu}(x)$ local with respect to $A(x)$, viz. ${ }^{1}$

$$
\left[A(x), j_{\mu}(y)\right]=0, \quad \text { for } \quad(x-y)^{2}<0 .
$$

locally conserved, viz.

transforming according to

$$
\frac{\partial j_{\mu}(x)}{\partial x_{\mu}}=0
$$

$$
U(\Lambda, a) j_{\mu}(x) U(\Lambda, a)^{-1}=\left(\Lambda^{-1}\right)_{\mu}^{\prime \nu} \cdot j_{\nu}(\Lambda x+a) .
$$

vi) The quantity ${ }^{2}$

exists and is self-adjoint

$$
Q=\int d^{3} x j_{0}\left(\boldsymbol{x}, x_{0}\right)
$$

$$
Q=Q^{+} \text {. }
$$

Then we have the

\section{Conjecture}

$$
Q=0 \text {. }
$$

Proof. From the relative locality of $A$ and $j_{\mu}$ follows that they have a common TCP operator and

$$
\Theta j_{\mu}(x) \Theta=-j_{\mu}(-x)
$$

From (17) and (14) follows then that

$$
\Theta Q \Theta=-Q \text {. }
$$

According to (12) $Q$ is time independent and in virtue of (14), (12) and (13) $Q$ commutes with $U(\Lambda, a)$. Let us introduce the fields

$$
B_{\alpha}(x) \equiv e^{i \alpha Q} A(x) e^{-i \alpha Q}, \quad \alpha \text { real number . }
$$

It sounds plausible that the one parameter group of unitary operators $e^{i Q \alpha}$ mapps local rings into local rings in virtue of (11). Even if the mapping in question would lead to a quasilocality, i.e. to

$$
R^{N}\left[A(x), B_{\alpha}(x+\boldsymbol{R})\right] \underset{R \rightarrow \infty}{\longrightarrow} 0, \quad N \text { any real integer }
$$

or almost-locality [see formula (6)] this would do for our purposes. A general proof of such a conjecture looks difficult (although there are

\footnotetext{
${ }^{1}$ From locality and irreducibility of $A(x)$ and from (11) follows the locality of $j_{\mu}(x)$.

2 A rigorous definition of $Q$ is given e.g. in $[1,2]$ and extensively discussed there. 
some statements of this sort which, however, take into account the structure of the current). Should nevertheless a current exist for which no one of the above mentioned requirements is fulfilled the symmetry induced by such a current can be hardly regarded as a physical one [9]. Let us accept in the following that $B_{\alpha}(x)$ is almost local with respect to $A(x)$. Then the premises of the Statement in $\S 2$ are satisfied. Therefore

$$
Q=a N_{\text {in }}+b=a^{\prime} N_{\text {out }}+b^{\prime}, \quad a, b, a^{\prime}, b^{\prime} \text { real numbers . }
$$

But $b=b^{\prime}=0$ since $Q \Omega=0$ which in turn follows from $\left(\Omega, j_{\mu}(x) \Omega\right)=0$. Further, from (20) we have $Q \psi_{s}=a \psi_{s}=a^{\prime} \psi_{s} ; \psi_{s} \in \mathscr{H}^{(1)}=$ subspace of one particle states (stability of the one particle states). Taking into account that the TCP operator mapps one particle states into one particle states and in virtus of (18) - we have $a=a^{\prime}=0$. This accomplishes the proof.

\section{Extension of the Conjecture to the Case of One Complex Field}

The procedure presented in $\S 3$ can be easily extended to the case of one complex scalar field. Consider one complex, scalar field $D(x)$ satisfying all beforementioned requirments including irreducibility of $D(x)$ and $D^{+}(x)$. To exclude trivial cases [e.g. $D(x)=e^{i \alpha} \varphi(x)$ where $\varphi(x)$ is hermitean] we assume

$$
a E\left(p^{2}=m^{2}, p_{0}>0\right) D(f) \Omega+b E\left(p^{2}=m^{2}, p_{0}>0\right) D^{+}(f) \Omega=0
$$

for $a=b=0$ only.

Then we construct a new field

$$
C(x)=\alpha D(x)+\beta D^{+}(x)
$$

where $\alpha$ and $\beta$ are chosen in such a way that

$$
\begin{aligned}
\left(\Omega, C(x) E\left(p^{2}\right.\right. & \left.\left.=m^{2}, p_{0}>0\right) C(y) \Omega\right)=0, \\
\left(\Omega, C(x) E C^{+}(y) \Omega\right) & =\left(\Omega, C^{+}(x) E C(y) \Omega\right) \\
& =i \Delta^{(+)}\left(x-y ; m^{2}\right)
\end{aligned}
$$

hold. $\mathscr{H}^{(1)}$ splits into two invariant subspaces carrying irreducible representations of the Poincaré group, spanned by $E C(f) \Omega$ and $E C^{+}(f) \Omega$ resp. Since $Q$ commutes with $U(\Lambda, a)$ it is a multiple of unity on every invariant subspace, viz.

$$
Q E C(f) \Omega=a E C(f) \Omega+b E C^{+}(f) \Omega .
$$

Notice that the TCP operator carries vectors from one invariant subspace into the other one and vice versa. Taking into account (18) and (15) we get from (23)

$$
\begin{gathered}
Q E C^{+}(f) \Omega=-a E C^{+}(f) \Omega \\
a-\text { a real number } .
\end{gathered}
$$


Similar considerations to that of $\S 3$ yield the asymptotic formula

where

$$
e^{i Q \alpha} C_{\text {out }}(y) e^{-i Q \alpha}=e^{i a \alpha} C_{\text {out }}(y)
$$

$C_{\mathrm{in}}(x)=\left(\frac{1}{2 \pi}\right)^{3 / 2} \int d k\left(B_{\mathrm{in}}^{+}(k) e^{i k x}+A_{\mathrm{in}}(k) e^{-i k x}\right) \delta\left(k^{2}-m^{2}\right) \Theta\left(k_{0}\right)$.

Taking into account the irreducibility of $C_{\text {in }}$ and $C_{\text {in }}^{+}$the unique solution for $(24)$ is

$$
Q=a\left\{N_{\text {out }}(B)-N_{\text {out }}(A)\right\}=a\left\{N_{\text {in }}(B)-N_{\text {in }}(A)\right\}
$$

where $N(B)$ is the number operator for the particles $B, N(A)$ - for particles $A$. Thus the only symmetry induced by a current in case of one irreducible, scalar, complex field can be the gauge group. There can be several, different currents giving rise to the same "charge" $Q$, e.g. the the "electric" currents $j_{\mu \text { in }}(x)$ and

$$
j_{\mu \text { out }}(x)=i a:\left(C_{\text {out }} \frac{\partial C_{\text {out }}^{+}}{\partial x_{\mu}}-\frac{\partial C_{\text {out }}}{\partial x_{\mu}} C_{\text {out }}^{+}\right):(x)
$$

$a$ real (which do not coincide in general). We leave the question open does there exist at least one such current, local with respect to $C(x)$. Notice that (26) does not imply the existence of a superselection rule (i.e. vanishing of Wightman functions constructed of different number of $C$ and $C^{+}$-fields).

\section{Remarks About the Equivalence of Similarity and Unitary Transformations}

Incidentally we show that a similarity transformation which links two real fields together can be practically always replaced by a unitary transformation [4]. More specifically, consider two real fields $A(x)$ and $B(x)$, transforming under the same representation of the translation group

$$
T(Q) \equiv U(I, a) .
$$

$A(x)$ being in addition cyclic with respect to the unique vacuum. Then we have the

\section{Statement. If}

and

$$
B(x)=\mathscr{V} A(x) \mathscr{V}-1
$$

then

$$
\mathscr{V} \Omega=\sigma \Omega, \quad \sigma \text { a number } \neq 0
$$

$$
B(x)=V A(x) V^{+}
$$

where $V$ is unitary and

$$
V \Omega=\Omega .
$$


Outline of the Proof. The first step in the proof consists in showing that $\mathscr{V}$ commutes with $T$. Exploiting this we show that $\mathscr{V}+\mathscr{V}=\mathscr{V} \mathscr{V}^{+}$ $=|\sigma|^{2}$. Then $V=\frac{1}{\sigma} \mathscr{V}$. Essential for the proof is that $\Omega$ is a separating vector for the commutant of the algebra of $A(x), \mathscr{V}$ has an inverse as well as reality of the fields and $(27 \mathrm{~b})$. However, no reference to locality and spectrality is made.

Should we make a stronger assumption requiring $A(x)$ to be irreducible we may weaken $(27 \mathrm{~b})$ to $(\Omega, \mathscr{V} \Omega) \neq 0$.

If we add to the original assumptions the spectrality condition we may drop the covariance of $B(x)$ under $T(a)$ and replace (27b) by $\|\mathscr{V} \Omega\| \neq 0$ and still get the result (28a) [but not $(28 \mathrm{~b})$ ].

We emphasize that the tensor transformation character of the fields $A$ and $B$ is irrelevant here since sofar we made use of translation invariance only.

\section{Final Remarks}

It is essential for our proofs in $\S 3$ and $\S 4$ that the symmetry is induced by a vector current. For instance for a tensor current (17) does no longer hold.

Should our conjecture, exhibited in $\S 3$ and $\S 4$, be true it does not exclude the existence of conserved real vector fields satisfying all the requirments listed in $\$ 3$ except (11) (relative locality). E.g. the only currents constructed out of bilinear combinations of a free field are

$$
\begin{aligned}
& \tilde{j}_{\mu}^{(1)}(k) \equiv\left(\frac{1}{2 \pi}\right)^{2} \int j_{\mu}^{(1)}(x) e^{i k x} d x \\
& =\iint\left(k_{1}-k_{2}\right)_{\mu}\left(\varepsilon\left(k_{1}\right)-\varepsilon\left(k_{2}\right)\right) C\left(k_{1} k_{2}\right) \delta\left(k-k_{1}-k_{2}\right) \\
& \quad \times \delta\left(k_{1}^{2}-m^{2}\right) \delta\left(k_{2}^{2}-m^{2}\right): \widetilde{A}_{0}\left(k_{1}\right) \widetilde{A}_{0}\left(k_{2}\right): d k_{1} d k_{2}
\end{aligned}
$$

or

$$
\tilde{j}_{\mu}^{(2)}(k)=a \int \Theta\left(k_{1}\right) k_{1, \mu} \delta\left(k_{1}^{2}-m^{2}\right) \widetilde{A}_{0}\left(-k_{1}\right) \widetilde{A}_{0}\left(k_{1}\right) d k_{1} \delta(k)
$$

or its linear combination. They satisfy all the assumptions except (11). Here

$$
C\left(k_{1} k_{2}\right)=\overline{C\left(k_{1} k_{2}\right)}, \quad a=\bar{a}, \quad \text { are } c \text {-numbers . }
$$

The special case of $(29)$ is $C\left(k_{1} k_{2}\right)=1$, viz.

$$
j_{\mu}(x)=i\left\{A_{0}^{(-)}(x) \frac{\partial A_{0}^{(+)}(x)}{\partial x_{\mu}}-\frac{\partial A_{0}^{(-)}(x)}{\partial x_{\mu}} A_{0}^{(+)}(x)\right\}
$$

up to a real constant. $j_{\mu}^{(2)}(x)$ is equal (up to a constant) to the energymomentum four vector $P_{\mu}$ (generators of the translation group). However, both currents, (29) and (30), annihilate the vacuum. Hence they can not be local with respect to any local real scalar irreducible field (according to the Reeh and Schlieder Theorem [10]) although $P_{\mu}$ is local with respect to itself $\left(j_{\mu}^{(1)}(x)\right.$ is not even local with respect to itself). 
Let us wind up the discussion with an example in favour of our conjecture

$$
\begin{aligned}
j_{\mu}^{(3)}(x)= & \left\{\left[\left(\frac{\partial}{\partial x_{1}}+\frac{\partial}{\partial x_{2}}+\cdots+\frac{\partial}{\partial x_{n}}\right)^{2} \frac{\partial}{\partial x_{1, \mu}}\right.\right. \\
& -\left(\frac{\partial^{2}}{\partial x_{1}^{2}}+\frac{\partial^{2}}{\partial x_{1} \partial x_{2}}+\cdots \frac{\partial^{2}}{\partial x_{1} \partial x_{n}}\right) \\
& \left.\cdot\left(\frac{\partial}{\partial x_{1}}+\frac{\partial}{\partial x_{2}}+\cdots+\frac{\partial}{\partial x_{n}}\right)_{\mu}\right] \\
& \cdot W\left(\frac{\partial^{2}}{\partial x_{1}^{2}}+\frac{\partial^{2}}{\partial x_{1} \partial x_{2}}+\cdots+\frac{\partial^{2}}{\partial x_{1} \partial x_{n}}\right)+\cdots \\
& +\left[\left(\frac{\partial}{\partial x_{1}}+\frac{\partial}{\partial x_{2}}+\cdots \frac{\partial}{\partial x_{2}}\right)^{2} \frac{\partial}{\partial x_{n, \mu}}\right. \\
& -\left(\frac{\partial^{2}}{\partial x_{1} \partial x_{n}}+\frac{\partial^{2}}{\partial x_{2} \partial x_{n}}+\cdots+\frac{\partial^{2}}{\partial x_{n}^{2}}\right) \\
& \left.\cdot\left(\frac{\partial}{\partial x_{1}}+\frac{\partial}{\partial x_{2}}+\cdots \frac{\partial}{\partial x_{n}}\right)_{\mu}\right] \\
& \left.\cdot W\left(\frac{\partial^{2}}{\partial x_{1} \partial x_{n}}+\frac{\partial^{2}}{\partial x_{2} \partial x_{n}}+\cdots \frac{\partial^{2}}{\partial x_{n}^{2}}\right)\right\} \\
& \cdot: A_{0}\left(x_{1}\right) A_{0}\left(x_{2}\right) \cdots A_{0}\left(x_{n}\right):\left.\right|_{x_{1}=x_{2}=\cdots=x_{n}=x}
\end{aligned}
$$

with $W(y)=\overline{W(y)}$ a polynomial ( $y$ is real), is local with respect to $A_{0}$, real, locally conserved and transforms under the same Poincaré representation as $A_{0}$. It can be easily shown by inspection that the "charge" vanishes since $\tilde{j}_{\mu}^{(3)}(k=0)=0$ and the current is locally conserved.

Acknowledgment. The author expresses his gratitude to H. Araki, G. F. Dell'Antonio, R. HaAq, A. JADCZYK, H. ReEH and A. Uhlmann for fruitful discussions and critical remarks.

Note Added in Proof. The Supplement of the Paper on "Symmetries Induced by Conserved Vector Currents in the Theory of a Scalar Field" by JAN T. ŁopUSZaŃsKI.

The conservation of the charge (26) and the symmetry generated by it do not directly imply the existence of a superselection rule [i.e. vanishing of the Wightman functions constructed out of unequal number of $C(x)$ and $C^{+}(x)$ fields]. However, one is able to construct another interacting irreducible field - let us call it $\varphi(x)$ - which satisfies already the requirments of the superselection rule and has as its asymptotic limits the free fields $C_{\mathrm{ex}}(x)$. The field $\varphi(x)$, however, does no longer need to be local; it is local, quasilocal or almostlocal depending on $C(x)$ being local quasilocal or almostlocal with respect to

$$
C^{\alpha}(x) \equiv e^{i Q \alpha} C(x) e^{-i Q \alpha} \quad \text { resp. }
$$


We have the

Statement. If $C(x)$ is (almost, quasi) local with respect to $C^{\alpha}(x)$ then the theory of a local complex interacting scalar field equipped with a symmetry generated by a conserved charge can be always replaced by a theory of another (almost, quasi) local, irreducible, interacting, complex scalar field satisfying the superselection rule requirments.

Outline of the Proof. Let us write

$$
e^{i \alpha Q}=\sum_{n=-\infty}^{+\infty} e^{i \alpha a n} F_{n} ; \quad \sum_{n=-\infty}^{+\infty} F_{n}=1,
$$

where $F_{n}$ is the projection operator on the subspace of charge $a n$. We have

$$
\sum_{n=-\infty}^{+\infty} F_{n+1} C_{\mathrm{ex}}(x) F_{n}=C_{\mathrm{ex}}(x)
$$

Let us introduce the complex field

$$
\varphi(x)=\sum_{n-=\infty}^{+\infty} F_{n+1} C(x) F_{n} .
$$

Since $Q$ commutes with $U(\Lambda, a)$ the field $\varphi(x)$ transforms under the same representation of the Poincaré as $C(x)$. We are going to show that $\varphi(x)$ is local, quasilocal or almostlocal depending on the locality relation between $C^{\alpha}(x)$ and $C^{\beta}(x)$ resp.

In case $C(x)$ and $C^{\alpha}(x)$ are local with respect to each other we have

$$
\begin{gathered}
F_{n+2} e^{i Q \alpha} C(x) e^{-i Q \alpha} C(y) F_{n}-F_{n+2} C(y) e^{i Q \alpha} C(x) e^{-i Q \alpha} F_{n}=0 \\
\text { for }(x-y)^{2}<0 .
\end{gathered}
$$

By multiplying this expression by $e^{-i a \alpha}$ and integrating it over $\alpha$ from $O$ to $2 \pi a^{-1}$ we get

$$
F_{n+2} C(x) F_{n+1} C(y) F_{n}-F_{n+2} C(y) F_{n+1} C(x) F_{n}=0
$$

or

$$
\varphi(x) \varphi(y)-\varphi(y) \varphi(x)=0 \text { for } \quad(x-y)^{2}<0 .
$$

In a similar way one gets also $\varphi(x) \varphi^{+}(y)-\varphi^{+}(y) \varphi(x)=0$ etc.

In case $C^{\alpha}(x)$ is quasilocal with respect to $C(x)$ the same procedure can be applied upon which a limit is taken, viz.

$$
\begin{gathered}
\lim _{R \rightarrow \infty} R^{N}\left[\varphi(x), \varphi\left(y^{0}, \boldsymbol{x}+\boldsymbol{R}\right)\right]=\frac{a}{2 \pi} \lim _{R \rightarrow \infty} R^{N} \int_{0}^{2 \pi / a} d \alpha e^{-i a \alpha} \quad(\mathrm{S} 6) \\
\sum_{n=-\infty}^{+\infty} F_{n+2}\left[C^{\alpha}(x), C\left(y^{0}, \boldsymbol{x}+\boldsymbol{R}\right)\right] F_{n}=\frac{a}{2 \pi} \int_{0}^{2 \pi / a} d \alpha e^{-i a \alpha} \sum_{n=-\infty}^{+\infty} F_{n+2} \\
\cdot\left(\lim _{R \rightarrow \infty} R^{N}\left[C^{\alpha}(x), C\left(y^{0}, \boldsymbol{x}+\boldsymbol{R}\right)\right]\right) F_{n}=0 \\
R \equiv|\boldsymbol{R}| \quad N-\text { integer number } .
\end{gathered}
$$


In case $C^{\alpha}(x)$ is almostlocal with respect to $C(x)$ we exploit the fact that $C^{\alpha}(x)$ is also almostlocal to $C^{\beta}(x)$. We compute the truncated functions of the fields $\varphi(x)$ and $\varphi^{+}(x)$ in the following way: e.g.

$$
\begin{aligned}
\left\langle\varphi\left(x_{1}\right)\right. & \left.\varphi\left(x_{2}\right) \varphi\left(x_{3}\right) \varphi^{+}\left(y_{1}\right) \varphi^{+}\left(y_{2}\right) \varphi^{+}\left(y_{3}\right)\right\rangle_{T} \\
= & \left(\frac{a}{2 \pi}\right)^{5} \int_{0}^{2 \pi / a} \cdots \int d \alpha d \beta d \gamma d \delta d \varepsilon e^{i a \alpha} e^{i \mathbf{2} a \beta} e^{i \mathbf{3} a \gamma} e^{i \mathbf{2} a \delta} e^{i a \varepsilon} \\
& \cdot\left(\Omega, C\left(x_{1}\right) C^{\alpha}\left(x_{2}\right) C^{\alpha+\beta}\left(x_{3}\right)\left(C^{\alpha+\beta+\gamma}\left(y_{1}\right)\right)^{+}\left(C^{\alpha+\beta+\gamma+\delta}\left(y_{2}\right)\right)^{+}\right. \\
& \left.\cdot\left(C^{\alpha+\beta+\gamma+\delta+\alpha}\left(y_{3}\right)\right)^{+} \Omega\right)-\Sigma\langle 4\rangle_{T}\langle 2\rangle_{T} .
\end{aligned}
$$

Generally, one gains the truncated functions of $l$ fields $\varphi(x)$ and of $k$ fields $\varphi^{+}(x)$ by integrating properly the truncated functions of the fields

and

$$
C^{\sum_{n=1}^{j-1} \alpha_{n}}\left(x_{j}\right), \quad j=1, \ldots, l
$$

$$
\left(C^{\sum_{n=1}^{l} \alpha_{n}+} \sum_{v=1}^{i-1} \alpha_{v+l}\left(y_{j}\right)\right)^{+}, \quad i=1, \ldots, k
$$

over $\alpha_{1}, \ldots, \alpha_{l+k}$. Hereupon it turns out that truncated functions of an odd number of fields $\varphi(x)$ and $\varphi^{+}(x)$ vanish. The exchange of the limit with the integration signs yields the desired result.

The (quasi, almost) locality together with $E \varphi(f) \Omega \neq 0$ assures the existence of the asymptotic fields

$$
\varphi_{\mathrm{ex}}(x)=C_{\mathrm{ex}}(x) .
$$

Since $C_{\text {ex }}(x)$ is assumed to be irreducible, $\varphi(x)$ and $\varphi^{+}(x)$ are also irreducible. Thus $C(x)$ can be expressed in terms of $\varphi(x)$ and $p^{+}(x)$. Moreover, $\varphi(x)$ is weakly local and weakly local with respect to $C(x)$ as it has the same TCP operator. It satisfies

$$
e^{i Q \alpha} \varphi(x) e^{-i Q \alpha}=e^{i \alpha a} \varphi(x) .
$$

This accomplishes the proof.

\section{References}

1. See e.g. Kastler, D., D. W. Robinson, and A. Swieca: Commun. Math. Phys. 2, 108 (1966).

2. See review articles on symmetries REEH, H.: Lecture Notes at the V-th Winter School of Theoretical Physics. Karpacz 1968. - Łopuszański, J.: Fortschr. Phys. 15, 681 (1967). - Robinson, D. W.: Lecture Notes at the NATO Summer School in Theoretical Physics. Instanbul 1966. CERN preprint 66/1205/5-Th. 708 .

3. Schroer, B., and P. Stichel: Commun. Math. Phys. 3, 258 (1966); - Swieca, A.: Nuovo Cimento, X, 52, 242 (1967).

4. Vassiliev, A.: Preprint, Kiev 1964. - Garczyński, W.: Bull. Acad. Polon. Sci. 14, 231 (1968); - Nuclear Phys. B 4, 689 (1968). 
5. For a definition of a free field see Streater, R. S., and A. S. Wightman: PCT, spin, statistics and all that. New York-Amsterdam 1964.

6. HaAG, R.: Phys. Rev. 112, 669 (1958). - Hepp, K.: Helv. Phys. Acta 37, 639 (1964); - Commun. Math. Phys. 1, 111 (1965). - Araki, H., and R. HaAg: Commun. Math. Phys. 4, 77 (1967).

7. Araki, H., R. HaAg, and B. Schroer: Nuovo Cimento X, 19, 90 (1961).

8. The original version due to Borchers, H. J.: Nuovo Cimento X, 15, 784 (1960); - was hereafter generalized by ARAKI, H.: see HEPP, K.: Lecture Notes of the Brandeis Summer School, 1965 as well as [7].

9. HaAG, R., and D. Kastler: J. Math. Phys. 5, 848 (1964).

10. Reet, H., and S. Schlieder: Nuovo Cimento, X, 22, 1051 (1961).

JAN T. ŁoPUSZAŃSKI

Inst. of Theoretical Physics

University of Wrocław

ul. Cybulskiego 36

Wrocław, Polen 